\title{
Auances en la Psicoterapia Analítica funcional
}

\author{
Aduances in functional Analytic Psychotherapy \\ Auanços na Psicoterapia Analítica Funcional
}

\author{
Rafael Ferro García \\ Centro de Psicología Clínica C.E.D.I. \\ Luis Valero Aguayo* \\ Universidad de Málaga
}

Doi: dx.doi.org/10.12804/apl33.01.2015.02

\section{Resumen}

El presente trabajo describe las variables que explican el proceso de cambio, los fundamentos y principios conductuales en los que se basa la Psicoterapia Analítica Funcional (FAP) y sus últimos avances. Sus fundamentos son los objetivos de intervención, es decir, las Conductas Clínicamente Relevantes (CCR) y también las cinco reglas terapéuticas propuestas. El análisis del proceso de cambio terapéutico propuesto permite comprender cuáles son las variables para conceptualizar un caso clínico. FAP hace hincapié en la relación cliente-terapeuta que da la oportunidad de moldear y reforzar las mejoras. Las líneas de investigación sobre eficacia y efectividad son prometedoras. Se describen recientes estudios de efectividad con diseños de caso único con medidas de la intervención con resultados positivos. Su integración con otras terapias de conducta de tercera generación produce efectos sinérgicos. Los avances expuestos de formas de evaluación y métodos de intervención dan oportunidades de innovar terapéuticamente y de evocar CCR necesarias para que se produzcan las mejoras.

Palabras Clave: Psicoterapia Analítica Funcional, relaciones cliente-terapeuta, procesos de cambio terapéuticos, avances

\section{flbstract}

This paper describes the variables that explain the process of change and foundations and behavioral principles that underpin Functional Analytic Psychotherapy (FAP) and its latest advances. Its foundations are the targets of intervention, that is, clinically relevant behaviors (CRB) and also the five proposed therapeutic rules. The analysis of the proposed therapeutic change process provides an insight into what variables to are necessary to conceptualize a clinical case. FAP emphasizes the client-therapist relationship which gives the opportunity to shape and reinforce improvements. The lines of research on efficacy and effectiveness are promising. We describe

* Rafael Ferro García, Centro de Psicología Clínica C.E.D.I.; Luis Valero Aguayo, Departamento de Personalidad, Evaluación y Tratamiento Psicológico de la Universidad de Málaga.

La correspondencia relacionada con este artículo debe ser dirigida a Rafael Ferro García, Avenida Constitución 25, $7^{\circ}$ Izqda. 18014 Granada, España. Correo Electrónico: rferro@cop.es

Para citar este artículo: Ferro, G. R., \& Valero, A. L., (2015). Avances en la Psicoterapia Analítica Funcional. Avances en Psicología Latinoamericana, 33(1), 15-30. doi: dx.doi.org/10.12804/ap133.01.2015.02 
recent studies of effectiveness of single-case designs of the intervention measures with positive results. Its integration with other third wave behavior therapies produces synergistic effects. The progress of the forms of evaluation and intervention described above provides opportunities for therapeutic innovations and to evoke necessary CCRs to bring about improvements.

Keywords: Functional Analytic Psychoterapy, client-therapist relationship, therapeutic change process, Advances

\section{Resumo}

O presente trabalho descreve as variáveis que explicam o processo de mudança, os fundamentos e princípios comportamentais nos que se baseia a Psicoterapia Analítica Funcional (FAP) e seus últimos avanços. Seus fundamentos são objetos de intervenção, é dizer, as Condutas Clinicamente Relevantes (CCR) e também, as 5 regras terapêuticas propostas. A análise do processo de mudança terapêutica proposta permite compreender quais são as variáveis para conceitualizar um caso clínico. FAP bate pé na relação cliente-terapeuta que dá a oportunidade de moldar e reforçar as melhoras. As linhas de pesquisa sobre eficácia e efetividade são prometedoras. Descrevem-se recentes estudos de efetividade com desenhos de caso único com medidas da intervenção com resultados positivos. Sua integração com outras terapias de conduta de terceira geração produz efeitos sinérgicos. Os avanços expostos de formas de avaliação e método de intervenção dão oportunidades de inovar terapeuticamente e de evocar CCR necessárias para que se produzam as melhoras.

Palavras-Chave: Psicoterapia Analítica Funcional, relações cliente-terapeuta; processos de mudança terapêuticas; Avanços

Hay un grupo de terapias que se han denominado como la tercera generación de la Terapia de Conducta (Hayes, 2004; O’Donohue, 1998; Pérez Álvarez, 2006). A este grupo pertenecen la Terapia de Conducta Dialéctica de Linehan (1993); la Psicoterapia Analitica Funcional (Kanter, Tsai \& Kohlenberg, 2010a; Kohlenberg \& Tsai, 1991; Tsai et al., 2009; Tsai, Kohlenberg, Kanter, Holman \& Loudon, 2012); la Terapia de Aceptación y Compromiso, ACT (Hayes \& Strosahl, 2004; Hayes, Strosahl \& Wilson, 1999); la Terapia Conductual Integrativa de Pareja (Jacobson \& Christensen, 1996) y la Activación Conductual (Jacobson, Martell \& Dimidjian, 2001; Martell, Adis \& Jacobson, 2001). Todas comparten los avances en el estudio de las relaciones de equivalencia entre los estímulos, en la conducta gobernada por reglas, en el análisis funcional del lenguaje y su repercusión en las conductas cognitivas y emocionales (Fernández-Parra \& Ferro-García, 2006).

FAP es una psicoterapia por sí misma y también puede ser combinada con cualquier terapia, lo que produce unos resultados sinérgicos (Kohlenberg et al., 2005). Además, es una terapia integradora en el sentido de que puede integrar ejercicios y/o metáforas de otras terapias sin perder su fundamento teórico (López-Bermúdez, Ferro \& Valero, 2010a; Tsai, Kohlenberg, Kanter \& Waltz, 2009). Se basa en los principios filosóficos del conductismo radical y asume que las causas de la psicopatología y su tratamiento están íntimamente enlazadas con las relaciones interpersonales (Baruch et al., 2009).

En este artículo se analizan las bases y los procesos de cambio terapéutico en la Psicoterapia Analítica Funcional (FAP) y también los recientes avances que han aparecido desde la publicación del primer manual (Kohlenberg \& Tsai, 1991).

\section{Fundamentos de FAP}

Los manuales de FAP (Kanter, Tsai \& Kohlenberg, 2010a; Kohlenberg \& Tsai, 1991; Tsai et al., 2009) no ofrecen un protocolo a seguir con fases, técnicas y procedimientos, sino que describe la forma de aplicar los principios básicos de su intervención. Por un lado, se describen los objetivos, las Conductas Clínicamente Relevantes (CCR) y, por otro, unas Reglas Terapéuticas a seguir. 
Las denominadas CCR (Kanter, Weeks et al, 2009; Kohlenberg \& Tsai, 1991, 1994a, 1994b, 1995; Kohlenberg, Tsai \& Kanter, 2009) serían todo el comportamiento del cliente y se diferencian tres tipos. Las CCR tipo 1 son los problemas del cliente que ocurren durante la sesión. Suelen estar bajo control de estímulos aversivos y son conductas de evitación. Pueden implicar pensamientos, percepciones, sentimientos, emociones, recuerdos, etc. Las CCR tipo 2 son las mejorías del cliente que ocurren durante la sesión. Las CCR tipo 3 son las interpretaciones del cliente de su propia conducta y lo que cree que la causa. Implican observaciones y descripciones de la conducta de uno mismo y de los estímulos reforzantes, discriminativos y elicitadores que están asociados con ella.

Se proponen unas Reglas Terapéuticas (Kohlenberg et al., 2005; Kohlenberg \& Tsai, 1994a, 1995; Tsai, Kohlenberg, Kanter \& Waltz, 2009), que ayudan a evocar, identificar, reforzar e interpretar las conductas del cliente. La Regla $\mathrm{N}^{\mathrm{o}} 1$ consiste en observar las posibles CCR durante la sesión terapéutica. La regla $\mathrm{N}^{\circ} 2$ propone construir un ambiente terapéutico que evoque las CCR. La regla $\mathrm{N}^{\circ} 3$ estriba en organizar el reforzamiento positivo de las CCR tipo 2. La regla $\mathrm{N}^{\circ} 4$ trata de desarrollar un repertorio para observar las propiedades reforzantes de la conducta del terapeuta en relación con las CCR del cliente. Finalmente, la regla $\mathrm{N}^{\circ} 5$ consiste en generar en el cliente un repertorio de descripción de las relaciones funcionales entre las variables de control y las conductas.

\section{El proceso de cambio terapéutico en FAP}

El proceso de cambio terapéutico en FAP podría resumirse en el esquema publicado por Ferro-García, Valero-Aguayo y López-Bermúdez (2009). Parte de la propuesta de la conceptualización de un caso (Kohlenberg, Kanter, Bolling, Parker \& Tsai, 2002; Kohlenberg \& Tsai, 2000; Kanter et al., 2009) que consiste en un registro abierto donde se describen las siguientes variables: los proble- mas que el cliente tiene en la vida diaria. Su historia relevante que está implicada en la etiología y el mantenimiento del problema. Los problemas que aparecen en la sesión clínica. Los conceptos cognitivos del paciente que interfieren con el tratamiento, es decir, pensamientos automáticos, código de creencias, valores, ideas, asunciones, etc. Sus objetivos de la vida diaria en función de sus propios valores. Y los objetivos que marcaría el terapeuta en la propia sesión. Pues bien, el proceso de cambio es un moldeamiento continuo por parte del terapeuta. Se establece una equivalencia funcional entre los problemas de la vida diaria y los problemas del cliente dentro de la sesión (CCR tipo 1). Es decir, si las variables que mantienen una conducta problemática en el contexto diario son las mismas que las que lo mantienen en la consulta, entonces las conductas funcionalmente son equivalentes (Kohlenberg, Tsai, Parker, Bowling \& Kanter, 1999). El terapeuta debe observar y evocar estas conductas (Reglas 1 y 2) y además, las mejorías del cliente dentro de la sesión clínica (CCR tipo 2) se generalizan a la vida diaria, por medio del reforzamiento natural y comprobar sus efectos en interacciones futuras (Reglas 3 y 4). La historia relevante cumple un papel clave en la génesis y el mantenimiento de los problemas de las personas, tanto en la vida diaria como en la propia sesión terapéutica. Por otro lado, las interpretaciones y explicaciones que tienen los clientes de lo que les ocurre (CCR tipo 3) pueden ser correctas o problemáticas (CCR 1 y 2) y suelen estar influenciadas por los conceptos cognitivos o ideas acerca de lo que les pasa, sobre qué creen que es la salud o el bienestar psicológico. Es necesario que el terapeuta genere en el cliente un repertorio de descripción de las relaciones funcionales entre las variables de control y las propias conductas (Regla 5) presentando modelos explicativos al cliente. La explicación dada por el terapeuta es un elemento importante en la propia explicación del cliente, es decir el tipo de razonamiento que se dé al cliente sobre la relación entre pensamientos y sentimientos en la terapia va 
a influir en los informes que den los clientes sobre su propio comportamiento (Kanter, Kohlenberg \& Loftus, 2002, 2004). Se puede concluir que las respuestas del cliente tienden a cambiar progresivamente según las contingencias que se apliquen y que el terapeuta debe adaptarse al lenguaje del cliente con un lenguaje cercano, tal y como se propone en los manuales de FAP (Kohlenberg \& Tsai, 1991; Tsai, Kohlenberg, Kanter \& Waltz, 2009).

Las Reglas de FAP se basan en unos principios conductuales aceptados y demostrados (Kanter, Tsai \& Kohlenberg, 2010b), como el control de estímulo (Regla 1 y 2), el reforzamiento (Regla 3 y 4) y la generalización (Regla 5).

\section{EI análisis funcional del lenguaje en FAP}

El análisis funcional del lenguaje, según la clasificación de Skinner (1957), es otra de las bases de FAP. El uso de los términos Tacto y Mando ayudan al terapeuta a tener conciencia de las CCR (Valero, Ferro, Kohlenberg \& Tsai, 2011). De una manera simple, un Tacto es una descripción y un Mando es un requerimiento o una petición. Analizar la conducta verbal del cliente en estos términos permite clasificar tales conductas y actuar según su función. Por ejemplo, un cliente se disculpa por llegar tarde a una cita diciendo "lo siento, por mucho que lo intento siempre llego tarde". En general, podría ser una mera descripción de la situación (un tacto), o tener una función de pedir disculpas (un mando) y/o evitar la posible contingencia aversiva del terapeuta (otro tipo de mando), tener una función de búsqueda de reforzamiento positivo o aprobación social, o quizás bien una petición o mando de ayuda por algún problema, incluso una petición de cambiar la hora de la consulta la próxima vez. También podría ser un desafío hacia la autoridad del terapeuta (mando disfrazado).

\section{Modelo explicativo del desarrollo del Yo propuesto por FAP}

El modelo propuesto por Kohlenberg y Tsai (1991, 2001) describe cómo se desarrolla el Yo y también explica los Trastornos de Personalidad y su tratamiento. El proceso de adquirir el informe verbal Yo es equivalente al aprendizaje de conceptos. El Yo como una unidad independiente surge de frases más largas previamente aprendidas que contenían ese Yo. Cuando se enseña a decir: "Yo veo un pájaro" o "Yo siento pena", hay una estimulación pública (los objetos, las señales externas de una emoción, llanto, los padres, etc.) y otra privada (estimulación interoceptiva, propioceptiva, etc.) y por medio de este moldeamiento que realizan los padres, la respuesta "Yo Veo X" pasa al control privado del niño. Es decir, la clave en este desarrollo radica en transferir el control de estas respuestas de lo público a lo privado. Este aprendizaje verbal se realiza por medio de tres etapas en las que el Yo como una unidad independiente surge de frases más largas, previamente aprendidas que contenían Yo.

El grado de dificultad experimentado por un individuo puede variar en función del grado de control privado sobre sus respuestas "Yo X" (quiero, siento, veo, etc.). En general, cuantas menos respuestas de este tipo se tengan bajo control privado, mayor será la confusión o dificultad al contestar preguntas que tengan que ver con sus preferencias personales, deseos, valores, etc. Los denominados problemas de personalidad tienen su origen en un aprendizaje poco adecuado de la formación del Yo, por contingencias de los padres poco estructuradas, por pérdida de control de la estimulación privada y/o contingencias que mantengan la discrepancia entre eventos privados y públicos. Las personas con Trastornos del Yo tienen un número significativo de respuestas del tipo "Yo X" bajo control público total o parcialmente (Kohlenberg \& Tsai, 1991) y su sentido del Yo se verá afectado. No es 
un problema de habilidad para exponer qué sienten o quieren, sencillamente ante esas personas no saben qué sienten, qué quieren, etc.

La intervención ante estos problemas se centra en reforzar las relaciones "Yo X" apropiadas en terapia. Es decir, reforzar que se hable en ausencia de indicaciones externas específicas, igualar las tareas terapéuticas al nivel de control privado del repertorio del cliente, reforzar todas las respuestas "Yo X" que surjan y usar técnicas de auto-observación y de toma de conciencia y de perspectiva (Kohlenberg \& Tsai, 1991, 2001; Kohlenberg, Tsai, Kanter \& Parker, 2009).

\section{Líneas de investigación actuales}

En una revisión reciente (Mangabeira, Kanter \& Del Prette, 2012) se mantiene que hay unos noventa autores diferentes que publican en FAP distribuidos en tres países, USA, Brasil y España. También se mantiene que ha habido un crecimiento exponencial en el número de publicaciones en los últimos diez años. Tras la publicación del monográfico sobre FAP en International Journal Of Behavioral Consultation And Therapy (2012, vol. 7, No 2-3), ha habido un incremento tanto en diferentes países (Colombia, Italia, Canadá, Reino Unido, Australia) como en el incremento de grupos de investigación en USA, fundamentalmente, y la colaboración entre grupos de diferentes países. Las líneas de investigación llevadas a cabo en FAP podrían resumirse en estudios aplicados y experimentales, formas de evaluación y conceptualización de casos, instrumentos de medida e intervención, procedimientos para mejorar y supervisar a los terapeutas, y su integración con otras terapias.

\section{Estudios aplicados y experimentales de FAP}

FAP se ha aplicado con éxito en pacientes con ansiedad (Kohlenberg \& Tsai, 1995; López-Bermúdez, Ferro-García \& Calvillo, 2010a; Vandenberghe
\& Martins, 2012); en pacientes con obsesiones (Kohlenberg \& Vandenberghe, 2007; Mendes y Vandenberghe 2009; Vandenberghe, 2007); en celos (Carrascoso, 2003); en clientes con depresión (Ferro-García, Valero \& Vives, 2006; Gaynor \& Scott, 2002; Holman, et al, 2012; Kanter, et al., 2006; Kohlenberg, et al., 2002; Kohlenberg \& Tsai, 1994a; López-Bermúdez, Ferro-García \& Valero, 2010b; McClafferty, 2012; Novoa \& Guzmán, 2008). También se ha aplicado en pacientes con trastornos de personalidad (Bastos, Kanter \& Meyer, 2012; Callaghan, Summers \& Weidman, 2003; Ferro-García, López-Bermúdez \& Valero, 2012; Koerner, Kohlenberg \& Parker, 1996; Manduchi \& Schoendorff, 2012; Vandenberghe \& Martins, 2012) y, más específicamente, en personas con trastorno de Personalidad Límite (Bastos, Kanter \& Meyer, 2012; Kohlenberg \& Tsai, 2000; Sousa, 2004). Se ha aplicado en terapia de pareja (Kohlenberg \& Tsai, 1995; Rabin, Tsai \& Kohlenberg, 1996). En clientes que mostraban anorgasmia (Oliveira \& Vandenberghe, 2005; Vandenberghe, Nasser, Silva \& Pereira, 2010). En sujetos que han sufrido abusos sexuales y estrés postraumático (Kohlenberg \& Tsai, 1998; Pedersen, Callaghan, Prins, Nguyen \& Tsai, 2012; Pergher \& Colombini, 2010; Prins \& Callaghan, 2002). En esquizofrenia paranoide (Bastos, Kanter \& Meyer, 2012). Se ha aplicado en problemas de salud, como en dolor crónico (Vandenberghe \& Ferro, 2005; Vandenberghe, Ferro \& Furtado, 2003; Vandenberghe, Furtado \& Ferro, 2003); en tabaquismo (Holman, et al., 2012); y en pacientes con fibromialgias (Queiroz \& Vandenberghe, 2006). Y en el ámbito infantil y juvenil, en un caso de un niño con conductas desafiantes (Gosch \& Vandenberghe, 2004); niños con déficits de aprendizaje (Xavier, Kanter \& Meyer, 2012); en el entrenamiento en habilidades sociales en pre-adolescentes (Cattivelli, Tirelli, Berardo \& Perini, 2012); y con jóvenes que han cometido delitos sexuales (Newring \& Wheeler, 2012).

En cuanto a las formas de aplicación, cabe resaltar los trabajos del equipo de Luc Vandenberghe 
(Vandenberghe, 2009; Vandenberghe \& Ferro, 2005; Vandenberghe, Ferro \& Furtado, 2003; Vandenberghe, Furtado \& Ferro, 2003) sobre la aplicación en grupo en problemas de depresión y dolor crónico, en los que presentan resultados positivos.

Los estudios sobre la efectividad y la eficacia hasta hoy son escasos pero esperanzadores. En el ensayo clínico más importante de FAP (Kohlenberg et al., 2002) participaron 46 sujetos repartidos en dos grupos, uno de 18 sujetos en un grupo de Terapia Cognitiva (Cognitive Therapy) y otro de 28 en un grupo denominado FECT (FAP Enhanced Cognitive Therapy) mejorada con FAP. Su duración fue de tres años y contó con la participación de tres terapeutas expertos en Terapia Cognitiva de Beck. En general, hubo una mejoría en el $79 \%$ de los participantes en el grupo de FECT, frente al $60 \%$ de los casos del grupo de CT de Beck. En un análisis posterior a los resultados (Kanter, Schildcrout \& Kohlenberg, 2005), el grupo FECT frente al grupo CT mejoraba variables terapéuticas como la cercanía, la adherencia y la satisfacción con el terapeuta. Además, los pacientes relacionaron más mejoras en la vida diaria, después de las intervenciones en vivo dentro de la terapia. Aunque sus resultados son buenos hay que indicar que este estudio presenta limitaciones metodológicas, como no ser un ensayo aleatorizado.

Callaghan, Summers \& Weidman (2003) presentan un estudio de efectividad por medio de los datos de un estudio de caso de un Trastorno de Personalidad Histriónico y Narcisista, donde se demuestran los cambios positivos producidos en el cliente a lo largo de la terapia y que son estadísticamente significativos.

Kanter et al. (2006) exponen dos casos clínicos que presentan Trastorno depresivo Mayor y Trastornos de Personalidad con un diseño cuasi-experimental. Los resultados indican que uno de los sujetos muestra una clara mejoría tras introducir las estrategias de FAP. En el otro, no hay cambios tan evidentes, ya que deja de registrar las conductas.
En los últimos tres años se ha producido un incremento de las publicaciones de intervenciones con diseños experimentales y cuasi-experimentales.

López-Bermúdez, Ferro y Valero (2010) presentan un estudio de caso del tratamiento de una mujer con Trastorno Depresivo Mayor, con un cambio significativo en las medidas de depresión pre-post, manteniéndose durante un seguimiento de 17 meses.

Xavier, Kanter y Meyer (2012) presentan una intervención con medidas repetidas a lo largo del tiempo, en dos casos de niños con dificultades de aprendizaje y uno de ellos con conductas desafiantes. Los resultados muestran que las conductas problemas disminuyen y las objetivo se incrementan en uno de ellos mientras que en el otro no llegan a eliminarse los problemas ni a estabilizarse las mejorías.

Ferro, López-Bermúdez y Valero (2012) exponen un estudio de caso de una mujer que presentaba un trastorno de Yo inestable tratado con FAP y donde se muestran unos cambios en los resultados de varias medidas pre-post, manteniendo los resultados en un seguimiento de 13 meses.

Bastos, Kanter y Meyer (2012) usan un diseño experimental de caso único en el que se replican las condiciones experimentales en dos pacientes, con unos resultados consistentes demostrando los cambios en las conductas de los clientes relacionados con las respuestas efectivas de los terapeutas.

Pedersen, Callaghan, Prins, Nguyen y Tsai (2012) usan FAP como tratamiento añadido tras tres años de un tratamiento estándar cognitivo-conductual en una mujer con estrés post-traumático. Los resultados muestran una clara mejoría después de la introducción de FAP, tanto en las medidas de los cuestionarios específicos como en la cercanía interpersonal y en la evitación.

Maitland y Gaynor (2012) han presentado una línea de investigación en curso comparando FAP con una situación de control como la escucha de 
apoyo, usando las relaciones interpersonales como variables dependientes y cuatro niveles de aplicación de FAP como variables independientes. Los datos que presentan son preliminares pero alentadores, muestran más beneficios a los sujetos del grupo FAP frente a una situación de control.

Holman, Kohlenberg y Tsai (2012) han desarrollado un protocolo de intervención breve de FAP y su primera aplicación en dos series de casos clínicos. En la primera serie se aplicó el protocolo en ocho personas que demandaron mejorar sus relaciones de pareja y los resultados indican que no hubo mejorías en las medidas estándar de satisfacción de las relaciones. En la segunda serie clínica, se corrigieron las deficiencias anteriores y se añadieron sesiones de supervisión del terapeuta y sesiones en grupo y cuestionarios de evaluación específicos. Se aplicó en otras seis personas y en este caso sí que hubo diferencias significativas en las medidas usadas.

Bowen, Haworth, Grow, Tsai y Kohlenberg (2012) exponen un ensayo piloto aleatorizado en tres grupos, en los que se compara una intervención breve basada en mindfulness en la que añaden aspectos interpersonales con FAP frente a meditación intrapersonal y a un grupo control. Los resultados indican que hubo diferencias significativas en la habilidad de conectar en la sesión y evitación experiencial del grupo interpersonal con FAP frente el grupo control y al de meditación. No hubo diferencias significativas en las medidas de seguimiento de intimidad, mindfulness y conectar con otros.

\section{Formas de evaluación}

Anteriormente, se describió una forma de conceptualizar un caso (Kanter et al., 2009). Además, Callaghan (2006a) ha elaborado otra forma conceptualizar un caso, llamada FIAT (Functional Idiographic Assessment Template). En ella se evalúan unas clases de comportamiento interpersonal que pueden ser problemáticas en los clientes. Estas clases son la exposición de las necesidades pro- pias, la comunicación bidireccional, la resolución de los conflictos, la cercanía interpersonal, la experiencia y la expresión emocional. Para cada una de ellas se establece una serie de cuestiones que el terapeuta debe plantearse. Se ha aplicado en varios casos demostrando ser útil (Callaghan, Summers \& Weidman, 2003; Kanter et al., 2009).

Por otro lado, se ha realizado un sistema de codificación de las conductas del cliente y del terapeuta, denominado The Functional Analytic Psychotherapy Rating Scale (FAPRS) (Callaghan, Summers \& Weidman, 2003). Este sistema permite obtener datos sobre los cambios que ocurren dentro de la sesión. La aplicación de este sistema ha demostrado su fiabilidad y utilidad para medir la efectividad de las terapias (Bastos, Kanter \& Meyer, 2012; Busch, Callaghan, Kanter, Baruch \& Weeks, 2010; Callaghan, Summers \& Weidman, 2003; Xavier, Kanter \& Meyer, 2012).

Se ha elaborado un cuestionario para evaluar la Experiencia del Yo (Experience of Self Scale, EOSS, Kanter, Parker \& Kohlenberg, 2001). Es un instrumento que pretende analizar y medir el grado de control público y privado sobre la experiencia del Yo. En la actualidad está siendo validado (Valero, Ferro, López-Bermúdez \& Selva, 2012; Valero, Ferro, López-Bermúdez, Selva \& Vives, 2011) con una muestra amplia, incluyendo población clínica. Los resultados señalan una alta fiabilidad y validez correlacionando significativamente con escalas que miden la despersonalización y la autoestima. También hay diferencias significativas entre la muestra normal y la clínica.

\section{Métodos terapéuticos evocativos}

En los últimos manuales se exponen nuevos métodos terapéuticos que sirven como herramientas de evaluación e intervención (Tsai et al., 2009). Se ha propuesto realizar un ejercicio experiencial de toma de conciencia y de toma de contacto profundo con el presente (mindfulness). En él, por medio de la respiración profunda, se provoca un estado 
de calma pasando por diferentes momentos de la historia personal (infancia, adolescencia, juventud, edad adulta), por diferentes comportamientos sociales, y con las personas significativas del cliente.

Se plantean una variedad de Ejercicios de Asociación Libre (Kohlenberg \& Tsai, 1991; Tsai, Kohlenberg, Kanter \& Waltz, 2009) como una manera de ayudar a personas que tienen Trastornos del Yo a poner bajo control privado tal experiencia. El propósito no es una forma de descubrir los contenidos ocultos sino que el objetivo es la propia conducta de asociar libremente, es decir, hablar en ausencia de control público de lo que sienten, piensan, etc. Se recomienda hacer los ejercicios con más o menos estructura dependiendo del cliente. El rango va desde decirle "Qué se te pasa por la cabeza cuando te digo esta serie de palabras...", hasta decirle: "Dime cualquier cosa que se te pase por la cabeza...". Una variedad de tipo de ejercicios es escribir en un ordenador lo primero que se ocurra sin ningún tipo de censura.

También se ha presentado un ejercicio para promocionar el mindfulness, el Ejercicio ARA (Awareness/Relaxation/Acceptance Exercise, Kohlenberg, Tsai, Kanter \& Parker, 2009) que permite estimular y reforzar la auto-observación, fomentando las habilidades de conciencia, relajación y aceptación. Es útil, como una manera de evocar CCR en estas situaciones de autoconciencia. Consta de tres fases, en un primer paso se asocia una palabra a la respiración profunda. Una segunda fase de observación y exploración muscular y respiración. Y, finalmente, una fase de aceptación y mindfulness, donde por medio de las fases anteriores se ayuda a afrontar las emociones y pensamientos temidos.

Hay una serie de ejercicios de escritura que pueden evocar CCR (Kohlenberg, Tsai, Kanter \& Parker, 2009). El Cuestionario FAP de Información Preliminar del Cliente es un auto-informe en el que se resumen los eventos vitales más importantes de su vida, una descripción breve de sí mismo y los objetivos que se marcaría en terapia. También se expone ejercicio de escritura con la mano no dominante, que consiste en una hoja de registro en la que se le pide al cliente que escriba con su mano no dominante sobre qué siente, qué necesita, qué miedos tiene, qué anhelos, etc. Se usa la mano no dominante porque esto le obliga a ser más breve y concreto. Su finalidad es marcar, de otra manera, los objetivos concretos en la terapia. Por otro lado, se plantea la Instantánea Vital de FAP, que es una manera rápida de valorar en forma de autoregistro, una variedad de áreas vitales (autocuidado, finanzas, amor, relaciones familiares, etc.).

\section{La supervisión de las habilidades terapéuticas}

Para mejorar las habilidades del terapeuta, Callaghan (2006b) ha creado la Functional Assessment of Skills for Interpersonal Therapists (FASIT). Esta escala evalúa y clasifica los problemas que el terapeuta puede tener durante el proceso de la terapia. Ha sido aplicada en varios estudios demostrando su utilidad (Callaghan, Gregg, Marx, Kohlenberg \& Gifford, 2004; Kohlenberg, Kanter, Bolling, Parker \& Tsai, 2002). Recientemente Schoendorff y Steinwatchs (2012) han propuesto el uso de FAP para entrenar terapeutas ACT aunque no presentan datos de este proyecto. Desde Brasil se han realizado dos experiencias de supervisión para psicólogos noveles en FAP y se describen los cambios que ha producido tal supervisión (Wielenska \& Bastos, 2012).

\section{Su integración con otras terapias}

FAP puede ayudar con sus principios a mejorar otras terapias. Se ha aplicado combinada con Terapia Cognitiva de Beck a lo que se denominó FAP - Enhanced Cognitive Therapy (Kohlenberg, Kanter, Tsai \& Weeks, 2010), y que se usó para realizar un ensayo clínico (Kohlenberg, et al., 2002), expuesto anteriormente. Por otro lado, Gaynor y Scott (2002) realizaron un estudio donde se integró FAP en la Terapia Cognitiva de Beck en el 
tratamiento de adolescentes depresivos. Los resultados indicaron que hubo una gran mejoría en los problemas depresivos manteniéndose durante un seguimiento de seis meses.

En la Universidad de Reno se ha aplicado junto con ACT, en lo que se ha denominado Functional-Analytic Acceptance and Commitment Therapy (FACT), en un programa para dejar de fumar (Callaghan, Gregg, Marx, Kohlenberg \& Gifford, 2004; Gifford et al., 2011). En este ensayo aleatorizado se comparó el bupropión frente a FACT junto a la medicación, mostrando que el tratamiento combinado da mejores resultados en dejar de fumar que la medicación sola, tanto al final del tratamiento como en el seguimiento a los seis meses y al año. También, se aplicó esta integración con ACT en el tratamiento de un caso de exhibicionismo obteniendo buenos resultados (Paul, Marx \& Orsillo, 1999). Esta combinación de ambas terapias se ha aplicado en el tratamiento de un cliente con depresión mayor y síntomas psicóticos presentando notables mejoras en depresión, aceptación y relaciones sociales (Baruch, Kanter, Busch \& Juskiewicz, 2009). Y también se integró en un Trastorno de Personalidad Obsesivo que presentaba anorexia nerviosa mostrando resultados positivos (Martín-Murcia, Cangas \& Pardo, 2011). Otra integración de estas terapias se ha aplicado a una adolescente que presentaba graves problemas en varias facetas de su vida de forma rígida obteniendo resultados positivos (Paéz \& Luciano, 2012). Novoa y Guzmán (2008) aplican principios y componentes de ACT y FAP en un joven con un trastorno mixto ansioso depresivo señalando los resultados y la utilidad de la intervención.

También se ha propuesto la integración de FAP en la Activación Conductual (AC) para mejorarla (Kanter, Manos, Busch \& Rusch, 2008). Esta combinación se ha aplicado en un estudio de caso mostrando unos buenos resultados (Manos et al., 2009). También McClafferty (2012) ha integrado $\mathrm{AC}$ con FAP en un caso de un hombre que presentaba un trastorno mixto ansioso depresivo. Los resultados de las medidas de los cuestionarios previo, durante y posterior a la intervención son más que significativos, llegando a la normalidad tras el tratamiento.

Holman et al. (2012) realizan un estudio piloto, donde han integrado Activación Conductual y Terapia de conducta clásica para dejar de fumar en el marco de FAP en cinco sujetos que presentaban depresión mayor y consumo de tabaco. Los resultados de los cambios pre-post en medidas de depresión indican que a cuatro de los cinco pacientes les remitió su depresión, y tres de los cinco sujetos dejaron de fumar completamente después del tratamiento. Finalmente Waltz, Landes y Holman (2010) analizan cómo FAP puede mejorar la Terapia de Conducta Dialéctica (DBT).

\section{Conclusiones}

En el presente trabajo se han descrito las variables que explican el proceso de cambio, los fundamentos y principios conductuales en los que se basa FAP y los últimos avances. La importancia dada por la terapia a las relaciones terapéuticas ha sido su mayor aportación a la psicoterapia en general. El análisis del proceso de cambio terapéutico propuesto permite ayudar al terapeuta a comprender cuáles son las variables claves para conceptualizar un caso clínico y actuar en concordancia con estas.

Hasta hace poco, la mayor crítica que se le hacía a FAP era el reducido número de publicaciones empíricas y la falta de estudios sobre eficacia y efectividad. Coincidimos con Maitland y Gaynor (2012) sobre las razones de por qué hay dificultad para generar investigaciones que soporten a FAP como tratamiento empíricamente validado. Un primer aspecto relevante es que es una aproximación general a la psicoterapia que no está basada en un modelo de psicopatología o no es específica para un determinado trastorno, lo que hace que sea difícil definir unas variables dependientes a los estudios. Además, su naturaleza idiosincrática y funcional y su amplia aplicabilidad hacen de FAP 
un reto para definir las variables independientes. Pues bien, desde una primera revisión teórica ( $\mathrm{Fe}-$ rro-García, 2008), hasta la actualidad, han pasado ya seis años, en los que las publicaciones y los estudios empíricos se han incrementado de forma exponencial. Una prueba de esto es la publicación de un monográfico sobre FAP en la revista antes citada, en el que hay más publicaciones empíricas que teóricas. También ha aumentado el número de grupos de investigación y grupos de nuevos países donde se estudia.

Las líneas de investigación sobre eficacia y efectividad son prometedoras aunque insuficientes. Son necesarios más estudios de eficacia donde se compare esta terapia con otras que son ya eficaces, con la no intervención y con el placebo, por medio de una metodología de grupo fiable con sólidos diseños de investigación experimental que ofrezcan datos cuantitativos sobre los efectos de esta intervención. Los recientes estudios de efectividad con diseños de caso único, tanto experimentales como cuasi-experimentales, con medidas de la intervención y de los resultados, han abierto una línea de investigación prometedora. Además, se sigue avanzando en la integración con otras terapias de esta generación y de la anterior. Pensamos que FAP sigue un buen camino para alcanzar los criterios para ser considerada un tratamiento validado empíricamente. Coincidimos con Mangabeira, Kanter y Del Prette (2012) en que se necesita un mayor diálogo y colaboración entre grupos de investigación de los diferentes países, como por ejemplo la línea abierta entre la Universidad de São Paulo y la Universidad de Wisconsin.

Los avances expuestos en las formas de evaluación y los métodos de intervención dan una oportunidad de innovar terapéuticamente y de evocar CCR necesarias para que se produzca el proceso de cambio. El uso de FAPRS como el sistema de codificación es útil para todo esto, siguiendo la línea de investigación abierta por Callaghan y colaboradores.
El incremento citado de publicaciones en los últimos años y la mejora en el control experimental de las investigaciones hace pensar que FAP goza de una buena salud y se puede pensar que hay un buen pronóstico en su futuro. En general, FAP es una terapia beneficiosa y rentable en el tratamiento de trastornos psicológicos, y que puede ayudar a los terapeutas a mejorar sus intervenciones.

\section{Referencias}

Baruch, D. E., Kanter, J. W., Busch, A. M., \& Juskiewicz, K. (2009). Enhancing the therapy relationship in Acceptance and Commitment Therapy for psychotic symptoms. Clinical Case Studies, 8, 241-257.

Baruch, D. E., Kanter, J. W., Busch, A. M., Plummer, M. D., Tsai, M., Rusch, L. C., Landes, S. J., \& Holman, G. I. (2009). Lines of evidence in support of FAP. En M. Tsai, R. J. Kohlenberg, J. W. Kanter, B. Kohlenberg, W.C. Follette, \& G. M. Callaghan, G. M. (Eds.), A Guide to Functional Analytic Psychotherapy. Awareness, Courage, Love and Behaviorism (pp. 21-36). New York: Springer.

Bastos, C. K., Kanter, J., \& Meyer, S. (2012). A single-case Experimental Demostration of Functional Analytic Psychotherapy with Two Clients with severe interpersonal problems. International Journal of Behavioral Consultation and Therapy, 7, 2-3. 111-116.

Bowen, S., Haworth, K., Grow, J. Tsai, \& Kohlenberg, R. J. (2012). Interpersonal Mindfulness Informed by Functional Analytic Psychotherapy: Finding from a Pilot Randomized Trial. International Journal of Behavioral Consultation and Therapy, 7(2-3), 9-15.

Busch, A. M., Callaghan, G. C., Kanter, J. W., Baruch, D. E., \& Weeks, C. E. (2010). The Functional Analytic Psychotherapy Rating Scale: A replication and extension. Journal of Contemporary Psychotherapy, 40, 11-19. 
Callaghan, G. M. (2006a). The Functional Idiographic Assessment Template (FIAT) System. The Behavior Analyst Today, 7, 357-398.

Callaghan, G. M. (2006b). The Functional Assessment of Skills for Interpersonal Therapists: The FASIT System. The Behavior Analyst Today, 7, 399-433.

Callaghan, G. M., Gregg, J. A., Marx, B. P., Kohlenberg, B. S., \& Gifford, E. (2004). FACT: The utility of an integration of Functional Analytic Psychotherapy and Acceptance and Commitment Therapy to Alleviate Human Suffering. Psychotherapy: Theory, Research, Practice, Training, 41, 195-207.

Callaghan, G. M., Summers, C. J., \& Weidman, M. (2003). The Treatment of Histrionic and Narcissistic Personality Disorder Behavior: A single-Subjetc Demostration of Clinical Improvement Using Functional Analytic Psychotherapy. Journal of Contemporary Psychotherapy, 33(4), 321-339.

Carrascoso, F. J. (2003). Jealousy: A case of application of Functional Analytic Psychotherapy. Psychology in Spain, 7, 88-98.

Cattivelli, R., Tirelli, V. Berardo, F., \& Perini, S. (2012). Promoting Appropriate Behavior in Daily Life Context Using Functional Analytic Psychotherapy in Early-Adolescent Children. International Journal of Behavioral Consultation and Therapy, 7(2-3), 25-32.

Fernández-Parra, A., \& Ferro-García, R. (2006). La Psicoterapia Analítico-Funcional: una aproximación contextual funcional al tratamiento psicológico. Edupsykhé, 5(2), 203-229.

Ferro-García, R. (2008). Recent Studies in Functional Analytic Psychotherapy. Internacional Journal of Behavioral Consultation and Therapy, 4(2), 239-249.

Ferro-García, R., López-Bermúdez, M. A., \& Valero-Aguayo, L. (2012). Treatment of a Disorder of Self through Functional Analytic Psychotherapy. Internacional Journal of Behavioral Consultation and Therapy. 7(2-3), 45-51.
Ferro-García, R., Valero-Aguayo, L., \& López-Bermúdez, M. A. (2009). La conceptualización de Casos Clínicos desde la Psicoterapia Analítica Funcional. Papeles del Psicólogo, 30(3), 255 264.

Ferro-García, R., Valero-Aguayo, L. \& Vives, M. C. (2006). Application of Functional Analytic Psychotherapy: Clinical Analysis of a patient with Depressive Disorder. The Behavior Analyst Today, 7, 1-18

Gaynor, S. T. \& Scott, P. (2002). Complementing CBT for depressed adolescents with learning through in vivo experience (LIVE): Conceptual Analysis, Trearment description, and feasibility study. Behavioral and Cognitive Psychotherapy, 30, 79-101.

Gifford, E. V., Kohlenberg, B. S., Hayes, S. C., Pierson, H. M., Piaseki, M. P., Antonnucio, D. O., \& Palm, K. M. (2011). Does acceptance and relationship focused behaviour therapy contribute to bupropion outcomes? A randomized controlled trial of functional analytic psychotherapy and acceptance commitment therapy for smoking cessation. Behavior Therapy, 42, 700-715.

Gosch, C. S., \& Vandenberghe, L. (2004). Behavior analysis and the therapist-child relationship in the treatment of an aggressive-defiant pattern. Revista brasileira de terapia comportamental e cognitiva, 6, 173-182.

Hayes, S. C. (2004). Acceptance and commitment therapy, relational frame theory, and third wave of behavioral and cognitive therapies. Behavior Therapy, 35, 639-665.

Hayes, S. C., \& Strosahl, K. D. (2004). A practical guide to Acceptance and Commitment Therapy. New York: Springer.

Hayes, S. C., Strosahl, K. D., \& Wilson, K. G. (1999). Acceptance and Commitment Therapy. An experimental approach to behaviour change. New York: Guilford Press.

Holman, G., Kohlenberg, R. J., \& Tsai, M. (2012). Development and Preliminary Evaluation of a FAP Protocol: Brief Relationship Enhancement. 
International Journal of Behavioral Consultation and Therapy, 7(2-3), 52-57.

Holman, G., Kohlenberg, R. J., Tsai, M., Haworth, Jacobson, E., \& Liu, S. (2012). Functional Analytic Psychotherapy is a Framework for Implementing Evidence-Based Practices: The example of Integrated Smoking Cessation and Depression Treatment. International Journal of Behavioral Consultation and Therapy, 7(23), 58-62.

Jacobson, N. J., \& Christensen, A. (1996). Integrative Couple Therapy: Promoting Acceptance and Change. New York: Norton.

Jacobson, N. S., Martell, C. R., \& Dimidjian, S. (2001). Behavioral activation treatment for depression: Returning to contextual roots. Clinical Psychology: Science and Practice, 8, 255-270.

Kanter, J. W., Kohlenberg, R. J., \& Loftus, E. F. (2002). Demand characteristics, treatment rationales, and cognitive therapy for depression. Prevention \& Treatment, 5, Article 41.

Kanter, J. W., Kohlenberg, R. J., \& Loftus, E. F. (2004). Experimental and psychotherapeutic demand characteristics and the cognitive therapy rationale: An analogue study. Cognitive Therapy and Research, 28(2), 229-239.

Kanter, J. W., Landes, S. J., Busch, A. M., Rusch, L. C., Brown, K. R., Baruch, D. E., \& Holman, G. (2006). The Effect of Contingent Reinforcement on Target Variables in Outpatient Psychotherapy for Depression: A Successful and Unsuccessful Case Using Functional Analytic Psychotherapy. Journal of Applied Behavior Analysis, 39, 463-467.

Kanter, J. W., Manos, R. C., Busch, A. M., \& Rusch, L. C. (2008). Making Behavioral Activation More Behavioral. Behavior Modification, 32(6), 780-803.

Kanter, J. W., Parker, C. \& Kohlenberg, R. J. (2001). Finding the self: A behavioral measure and its clinical implications. Psicotherapy: Theory, Research and Practice, 38, 198-211.
Kanter, J. W., Schildcrout, J. S., \& Kohlenberg, R. J. (2005). In vivo processes in cognitive therapy for depression: Frecuency and benefits. Psychotherapy Research, 15(4), 366-373.

Kanter, J. W., Tsai, M., \& Kohlenberg, R. J. (2010a). The Practice of Functional Analytic Psychotherapy. New York: Springer.

Kanter, J. W., Tsai, M., \& Kohlenberg, R. J. (2010b). Introduction to the Practice of Functional Analytic Psychotherapy. En J. W. Kanter, M. Tsai \& R. J. Kohlenberg (Eds.), The Practice of Functional Analytic Psychotherapy (pp. 1-7). New York: Springer.

Kanter, J. W., Weeks, C. E., Bonow, J. T., Landes, S. J., Callagahn, G. M., \& Follette, W. C. (2009). Assessment and Case Conceptualization. En M. Tsai, R. J. Kohlenberg, J. W. Kanter, B. Kohlenberg, W. C. Follette, \& G. M. Callaghan (Eds.), A Guide to Functional Analytic Psychotherapy. Awareness, courage, Love, and Behaviorism (pp. 37-59). New York: Springer.

Koerner, K., Kohlenberg, R. J., \& Parker, R. (1996). Diagnosis of Personality Disorder: A Radical Behavioral alternative. Journal of Consulting and Clinical Psychology, 64, 1169-1176.

Kohlenberg, R. J., Kanter, J. W., Bolling, M. Y., Parker, C. R., \& Tsai, M. (2002). Enhancing Cognitive Therapy for Depression with Functional Analytic Psychotherapy: Treatment Guidelines and Empirical Findings. Cognitive and Behavioral Practice, 9, 213-229.

Kohlenberg, R. J., Kanter, J. W., Tsai, M., \& Weeks, C. E. (2010). FAP and Cognitive Behavior Therapy. En J. W. Kanter, M. Tsai \& R. J. Kohlenberg (Eds.), The Practice of Functional Analytic Psychotherapy (pp. 11-30). New York: Springer.

Kohlenberg, R. J., \& Tsai, M. (1991/2008). Functional Analytic Psychotherapy. Creating intense and curative therapeutic relationship. New York: Plenum Press. (Traducción Servicio de Publicaciones Universidad de Málaga). 
Kohlenberg, R. J., \& Tsai, M. (1994a). Improving cognitive therapy for depression with functional analytic psychotherapy: Theory and case study. The Behavior Analyst, 17, 305-319.

Kohlenberg, R. J., \& Tsai, M. (1994b). Functional Analytic Psychotherapy: A Radical Behavioral Approach to Treatment and Integration. Journal of Psychotherapy Integration, 4, 174-201.

Kohlenberg, R. J., \& Tsai, M. (1995). Functional analytic psychotherapy: a behavioral approach to intensive treatment. En W. O’Donohue \& L. Krasner (Eds.), Theories of behavior therapy. Exploring behavior change (pp. 637-658).Washington: APA.

Kohlenberg, R. J., \& Tsai, M. (1998). Healing interpersonal trauma with the intimacy of the relationship. En V. M. Follette, J. I. Ruzeg \& F. R. Abueg (Eds.), Cognitive-Behavioral Therapies for Trauma (pp. 305-320). New York: Guilford.

Kohlenberg, R. J., \& Tsai, M. (2000). Radical Behavioral Help for Katrina. Cognitive and Behavioral Practice, 7, 500-505.

Kohlenberg, R. J., \& Tsai, M. (2001). Hablo, luego existo: una aproximación conductual para entender los problemas del yo. Escritos de Psicología, 5, 58-62.

Kohlenberg, R. J., Tsai, M., Ferro-García, R., Valero, L., Fernández Parra, A., \& Virués-Ortega, J. (2005). Psicoterapia analítico-funcional y terapia de aceptación y compromiso: Teoría, aplicaciones y continuidad con el análisis del comportamiento. International Journal of Clinical and Health Psychology, 5(2), 37-67.

Kohlenberg, R. J., Tsai, M., \& Kanter, J. W. (2009). What is Functional Analytic Psychotherapy? En M. Tsai, R. J. Kohlenberg, J. W. Kanter, B. Kohlenberg, W.C. Follette, \& G. M. Callaghan, G. M. (Eds), A Guide to Functional Analytic Psychotherapy. Awareness, Courage, Love and Behaviorism (pp. 1-19). New York: Springer.

Kohlenberg, R. J., Tsai, M., Kanter, J. W., \& Parker, C. R. (2009). Self and Mindfulness. En M. Tsai, R. J. Kohlenberg, J. W. Kanter, B. Kohlenberg,
W.C. Follette, \& G. M. Callaghan, G. M. (Eds.). A Guide to Functional Analytic Psychotherapy. Awareness, Courage, Love and Behaviorism (pp. 103-130). New York: Springer.

Kohlenberg, R. J., Tsai, M., Parker, C. R., Bolling, M. W., \& Kanter, J. W. (1999). Focusing on the client-therapist interaction: FAP a behavioral approach. European Psychotherapy, 1(1), 15-25.

Kohlenberg, R. J., \& Vandenberghe, L. (2007). Treatment resistant $\mathrm{OCD}$, inflated responsibility, and the therapeutic relationship: Two case examples. Psychology and Psychotherapy-Theory Research and Practice, 80, 455-465.

Linehan, M. M. (1993). Cognitive-Behavioral Treatment of Borderline Personality Disorder. New York: Guilford Press

López-Bermúdez, M. A., Ferro-García, R., \& Calvi1lo, M. (2010a). An Application of Functional Analytic Psychotherapy In a case of Anxiety Panic Disorder Without Agoraphobia. Internacional Journal of Behavioral Consultation and Therapy, 6(4), 356-372.

López-Bermúdez, M. A., Ferro-García, R., \& Valero, L. (2010b). Intervención en un Trastorno depresivo mediante la Psicoterapia Analítica Funcional. Psicothema, 22(1), 92-98.

Maitland, D. W. M., \& Gaynor, S. T. (2012). Promoting Efficacy Research on Functional Analytic Psychotherapy. International Journal of Behavioral Consultation and Therapy, 7(2-3), 63-71.

Manduchi, K., \& Schoendorff, B. (2012). First Steps in FAP: Experiences of Beginning Functional Analytic Psychotherapy Therapist with an Obsessive-Compulsive Personality Disorder Client. International Journal of Behavioral Consultation and Therapy, 7(2-3), 72-77.

Mangabeira, V., Kanter, J., \& Del Prette, G. (2012). Functional Analytic Psychotherapy (FAP): A review of publications from 1990 to 2010. International Journal of Behavioral Consultation and Therapy, 7(2-3), 78-89.

Manos, R. C., Kanter, J. W., Rusch, L. C., Turner, L. B., Roberts, N. A., \& Busch, A. M. (2009). 
Integrating Functional Analytic Psychotherapy and behavioral activation for the treatment of relationship distress. Clinical Case Studies, 8 , 122-138.

Martell, C. R., Addis, M. E., \& Jacobson, N. S. (2001). Depression in context: Strategies for guided action. New York: W.W. Norton.

Martín-Murcia, F., Cangas Díaz, A. J., \& Pardo González, L. (2011). A Case Study of Anorexia Nervosa and Obsessive Personality Disorder Using Third-Generation Behavioral Therapies, Clinical Case Studies, 10(3), 198-209.

McClafferty, C. (2012). Expanding the Cognitive Behavioural Therapy Traditions: An Application of Functional Analytic Psychotherapy Treatment in a Case Study of Depression. International Journal of Behavioral Consultation and Therapy, 7(2-3), 90-95.

Mendes, A. C., \& Vandenberghe, L. (2009). O relacionamento terapeuta-cliente no tratamento do transtorno obsessivo compulsivo. [The therapist-client relationship in the treatment of obsessive compulsive disorder]. Estudos de Psicologia (Campinas), 26, 545-552.

Newring, K. A. B., \& Wheeler, J. G. (2012). Functional Analytic Psychotherapy with Juveniles who have Committed Sexual Offenses. International Journal of Behavioral Consultation and Therapy, 7(2-3), 102-110.

Novoa Gómez, M. M., \& Guzmán Guitérrez, D. (2008). Aplicación clínica de modelos terapéuticos no mediacionales en un caso de trastorno mixto del afecto. Terapia Psicológica, 26(2), 263-275.

O'Donohue, W. (1998). Conditioning and third-generation behavior therapy. In W. O'Donohue (Ed.). Learning and Behavior Therapy (pp. 1-14). Needham Heights: Allyn and Bacon.

Oliveira, K. C. F., \& Vandenberghe, L. (2005). Anorgasmia e esquiva experiencial, um estudo de caso. Psicología Clínica, 17(1), 162-176.

Paéz, M., \& Luciano, C. (2012). La Integración de ACT y FAP en el trabajo con patrones rígidos de comportamiento. En M. Paéz \& O. Guitiérrez (Coords.). Múltiples aplicaciones de la Terapia de Aceptación y Compromiso (ACT). (pp. 4772). Madrid: Pirámide.

Paul, R.H.; Marx, B. P., \& Orsillo, S. M. (1999). Acceptance-based psychotherapy in the treatment of an adjudicated excibitionist: a case example. Behavior Therapy, 30, 149-162.

Pedersen, E. R., Callaghan, G. M., Prins, A., Nguyen, H., \& Tsai, M. (2012). Functional Analytic Psychotherapy as an Adjunct to Cognitive-Behavioral Traetments for Posttraumatic Stress Disorder: Theory and Application in a Single Case Design. International Journal of Behavioral Consultation and Therapy, 7(2-3), 125134.

Pérez Álvarez, M. (2006). La Terapia de Conducta de tercera generación. EduPsykhé, 5(2), 159-172.

Pergher, N. K., \& Colombini, F. A. (2010). Client's Revelations in Therapy Sessions. Acta Comportamentalia, 18(3), 361-380.

Prins, A., \& Callaghan, G. M. (2002). Functional Analytic Psychotherapy on an adjunctive treatment for a client meeting criteria for PSTD. Poster presentado en el 36 Annual Meeting of the Association for the Advancement of Behavior Therapy, Reno, November.

Queiroz, M. A. M., \& Vandenberghe, L. (2006). Psychotherapy in the treatment of fibromialgia: Interweaving FAP and ACT. In H. J. Guilhardi, \& N. Aguire. (Eds.), Sobre Comportamento e Cognição (pp. 238-248). Santo André: ESETEC.

Rabin, C., Tsai, M., \& Kohlenberg, R. J. (1996). Targeting sex-role and power issues with a functional analytic approach: Gender patterns in behavioral marital therapy. Journal of Feminist Family Therapy, 8, 1-24.

Schoendorff, B., \& Steinwachs, J. (2012). Using Functional Analytic Therapy to train therapists in Acceptance and Commitment Therapy, a conceptual and practical framework. Internacional Journal of Behavioral Consultation and Therapy.7(2-3), 135-137. 
Skinner, B. F. (1957/1981). Verbal behavior. Acton, MA: Copley Publishing. (Traducción: Ed. Trillas).

Sousa, A. C. A. (2004). Borderline personality disorder from a functional analytic perspective. Revista brasileira de terapia comportamental e cognitiva, 5, 121-137.

Tsai, M., Kohlenberg, R. J., Kanter, J. W., Holman, G. I. \& Loudon, M. P. (2012). Functional Analytic Psychotherapy. Distinctive Features. New York: Routledge.

Tsai, M., Kohlenberg, R. J., Kanter, J. W., Kohlenberg, B., Follette, W. C., \& Callaghan, G. M. (2009). A Guide to functional Analytic Psychotherapy. Awareness, Courage, Love, and Behaviorism. New York: Springer.

Tsai, M., Kohlenberg, R. J., Kanter, J. W., \& Waltz, J. (2009). Therapeutic Technique: The Five Rules. In M. Tsai, R. J. Kohlenberg, J. W. Kanter, B. Kohlenberg, W. C. Follette, \& G. M. Callaghan (Eds), A Guide to functional Analytic Psychotherapy. Awareness, courage, Love, and Behaviorism (pp. 61-102). New York: Springer.

Valero-Aguayo, L., Ferro-García, R., Kohlenberg, R. J., \& Tsai, M. (2011). Therapeutic Change Processes in Functional Analytic Psychotherapy. Clínica y Salud, 22(3), 209-221.

Valero-Aguayo, L., Ferro-García, R., López-Bermudez, M. A., Selva, M. A., \& Vives, M. C. (2011). Reliability and validity of the Spanish adaptation of Experiencing of Self Scale (EOSS) for evaluation in Functional Analytical Psychotherapy: First data with standard and clinical samples. Paper presented at 6Th Conference Association for Behavior Analysis International. Granada (Spain).

Valero-Aguayo, L., Ferro-García, R., López-Bermudez, M. A., \& Selva, M. A. (2012). Reliability and validity of the Spanish adaptation of Experiencing of Self Scale (EOSS) comparing normal and clinical samples. Internacional Journal of Behavioral Consultation and Therapy.7(2-3), 151-158.

Vandenberghe, L. (2007). Functional analytic psychotherapy and the treatment of obsessive compulsive disorder. Counseling Psychology Quarterly, $20,105-114$

Vandenberghe, L. (2009). A functional analytic approach to group therapy. The Behavior Analyst Today, 10(1), 71-82.

Vandenberghe, L., \& Ferro, C. L. B. (2005). Terapia de grupo embasada em psicoterapia analítica funcional como abordagem terapêutica para dor crônica: possibilidades e perspectivas. Psicologia: Teoria e prática, 7, 137-152.

Vandenberghe, L., Ferro, C. B. L., \& Furtado da Cruz, A. C. (2003). FAP-enhanced group therapy for chronic pain. The Behavior Analyst Today, 4, 369-375.

Vandenberghe, L., Furtado da Cruz, A. C., \& Ferro, C. B. L. (2003). Terapia de grupo para pacientes com dor cronica orofacial. Revista Brasileira de Terapia Comportamental e Cognitiva, 5(1), 31-40.

Vandenberghe, L., \& Martins da Silveira, J. (2012). The Trouble with the Short-Term Therapist-Clinete Relationship and What Can Be Done about it. Internacional Journal of Behavioral Consultation and Therapy, 7(2-3), 159-166.

Vandenberghe, L., Nasser, D. O., Silva, K. C. F. A., \& Pereira, D. (2010). Couples therapy, female orgasmic disorder and the therapist-client relationship: Two case studies in functional analytic psychotherapy. Counseling Psychology Quarterly, 23(1), 45-53.

Waltz, J., Landes, S. J., \& Holman, G. I. (2010). FAP and Dialectical Behavior Therapy (DBT). En J. M. Kanter, M. Tsai, \& R. J. Kohlenberg (Eds.), The Practice Of Functional Analytic Psychotherapy (pp. 47-64). New York: Springer.

Wielenska, R. C., \& Bastos, C. K. (2012). FAP Group Supervision: Reporting Educational Experiences at the University of São Paulo, Brazil. In- 
ternacional Journal of Behavioral Consultation and Therapy.7(2-3), 177-181.

Xavier, R. N., Kanter, J., \& Meyer, S. B. (2012). Transitional Probability Analysis of two Child Behavior Analytic Therapy Cases. International Journal of Behavioral Consultation and Therapy, 7(2-3), 182-188.

Fecha de recepción: 11 de diciembre de 2013 fecha de aceptación: 31 de Julio de 2014 